note (loc. cit., August) that Prof. Morse's latest corrected result (his own corrections) for the osmotic pressure at $0^{\circ} \mathrm{C}$. of his $\mathrm{r} \cdot \mathrm{O}$ weight normal aqueous solution of cane sugar is 24.45 atmospheres, while ours, by the method under discussion, was 24.5 atmospheres.

Evian les Bains.

BERKELEy.

\section{The Rotation of a Crystal of Tourmaline by Plane Polarised Light.}

\section{(Preliminary Note.)}

WHEN a beam of plane-polarised light is incident normally on a plate of tourmaline cut parallel to the optic axis, it will be absorbed or transmitted depending upon whether the axis of the tourmaline is parallel or perpendicular to the plane of polarisation of the incident light. If the arrangement is such that the light is absorbed, then in a given time a definite amount of heat energy will have passed from the source of light into the plate of tourmaline, and if, as is necessarily true, the former is at a high temperature while the latter is at a low temperature, it is plain that the entropy of the system will have been increased. The same increase in entropy would not have taken place if the orientation of the tourmaline had been such that the light had been transmitted.

Since the entropy of a system always tends to increase, it seemed to be of some interest to try what would happen if a plate of tourmaline was suspended so as to be free to rotate about an axis perpendicular to its faces, and then allowing a beam of plane-polarised light to fall on it in the direction of the axis of rotation, the arrangement being such that the plane of polarisation should make an angle of $45^{\circ}$ with the optic axis of the tourmaline.

The experiment was tried by the author during the month of July, 1908, at the Rouss Physical Laboratory of the University of Virginia, and the results, though not absolutely conclusive, indicate that a moment acts on the tourmaline tending to set its optic axis parallel to the plane of polarisation of the incident light. In other words, the system tends to arrange itself so that as large a percentage of the light as possible shall be absorbed.

The apparatus used consisted of a fine plate of tourmaline, $\mathrm{I} \mathrm{cm}$. square, and $2^{*} \cdot 96 \mathrm{~mm}$. thick, weighing almost exactly one gram. This was fastened to one end of a short straight copper wire to which was also fastened a small plane mirror. The system was suspended in a suitable vessel having a plane glass top and bottom, which could be exhausted, the suspension being such that the system had a period of $29^{\circ} 5$ seconds. From the period and the moment of inertia of the system it was calculated that the moment necessary to give a deflection of $\mathrm{I} \mathrm{cm}$. with the scale at a distance of one metre was $2.5 \times 10^{-5}$ dyne-cm. A beam of approximately parallel light from an arc was reflected in a vertical direction from a plane silver mirror, and was rendered plane polarised by a Nicol before entering the vessel.

Owing to the fact that the suspended system was not perfectly symmetrical with respect to its axis cf rotation, it was found that the zero moved constantly in one direction when the light was allowed to fall on the tourmaline. This was undoubtedly due to radiometric action, and possibly also to the pressure of light. The motion was, however, much slower when the angle between the plane of polarisation and the optic axis of the crystal was $+45^{\circ}$ than when it was $-45^{\circ}$. An average of twenty trials gave $15^{\circ}$ seconds as the time to get a deflection of $30 \mathrm{~cm}$. in the first case, while the time required for the same deflection when the angle was $-45^{\circ}$ was 90 seconds. These trials were all made with as high a vacuum in the vessel as could be obtained by means of the Gaede rotating mercury pump. The experiment was repeated at various pressures up to ro or $15 \mathrm{~cm}$. of mercury; the results were qualitatively the same, that is, the rate of deflection was much slower in every case when the angle mentioned above was $+45^{\circ}$ than when it was $-45^{\circ}$, but the motion of the zero in one direction, although somewhat different at different pressures, could not be avoided.

If the cause of this motion of the zero is what was stated above, then it ought to disappear when the sus- pended system is made symmetrical about the axis of rotation.

These experiments were only preliminary, and during the coming winter a more careful investigation of the question will be carried out at the physical laboratory of the Johns Hopkins University. Physical Laboratory, Johns Hopkins University, August ${ }_{15}$.

\section{Access to Chemical Works.}

Students of chemistry so often complain of the extreme difficulty of obtaining access to chemical works that it may be well to bear in mind that the universities and technical schools of the United Kingdom have perhaps a remedy for what is a serious obstacle to a proper study of chemistry. The amount of chemicals now consumed in educational laboratories must be enormous, and, as regards ordinary materials, quite sufficient to employ a large factory. A very large proportion comes from Germany. Let the universities start their own cooperative factory for the manufacture of the acids and salts they require. Let chemical students both have free access to it and put in part of their chemical course as workers there. The action of several universities in running their own farms for the benefit of agricultural students supplies a kind of precedent. When motives of education, patriotism, and economy point the same way, the scheme is worth consideration.

Chemist.

\section{FLY FEVER IN AFRICA.}

$\mathrm{IN}$ the Times of June 27 there appeared an article on "fly fever" in Africa, and the suggested destruction of big game. It appears that Prof. Koch has lately suggested that the African big game should be exterminated in order to destroy the principal means of nourishment of the tsetse fly. As this fly is the carrier of the infective agent of "fly fever," its extinction would, in Koch's opinion, blot out the disease. The members of the German Society for the Preservation of Game in East Africa are, naturally, opposing tooth and nail this proposed drastic measure of Prof. Koch. They deny the truth of his conclusions, and hold that the fly disease can exist where there is no big game, and, further, that there are other methods of getting rid of the fly without destroying the game.

This question is a complicated one, and perhaps if I give a short sketch of the history of the "tsetsefly disease "I shall best clear the way to its better understanding.

When I went to Zululand in 1894 at the request of the Natal Government, to investigate an outbreak of nagana among native cattle, I was unaware that nagana and tsetse-fly disease were one and the same. Further, I believed, with the rest of the world, that the fly disease was caused by the poison of the tsetse fly, just as an animal is killed by the poison of a snake. Soon after arriving in Zululand a parasite, the Trypanosoma brucei, was discovered in the blood of the affected animals, and it soon became apparent that fly disease and nagana were one and the same. By suitable experiments it was demonstrated that this trypanosome is the cause of the disease. The next fact made out was that the- trypanosome could be conveyed from sick to healthy animals by the local species of tsetse fly (Glossina morsitans). As this tsetse fly only remained infective for forty-eight hours, it was evident that it must get the parasite somewhere, and it seemed most probable that this would be from the wild animals living in the "fly country." This was found to be the case, and the Trypanosoma bruce $i$ was demonstrated in the blood of the buffalo, wildebeeste, koodoo, and other big game, both microscopically and by means of inoculation experiments.

NO. 2027 , VOL. $7 \mathrm{~S}]$ 